\title{
The UK Paediatric Ocular Trauma Study I (POTSI): development of a global standardized protocol for prospective data collection in pediatric ocular trauma
}

\author{
This article was published in the following Dove Press journal: \\ Clinical Ophthalmology \\ 23 February 2017 \\ Number of times this article has been viewed
}

\author{
Freda $\mathrm{Sii}^{1,2}$ \\ Robert J Barry' \\ Richard J Blanch' \\ Joseph Abbott ${ }^{3}$ \\ Caroline J MacEwen ${ }^{4}$ \\ Peter Shah ${ }^{1,2,5,6}$ \\ 'Department of Ophthalmology, \\ Queen Elizabeth Hospital Birmingham, \\ ${ }^{2}$ Birmingham Institute for Glaucoma \\ Research, Institute of Translational \\ Medicine, University Hospitals \\ Birmingham NHS Foundation Trust, \\ ${ }^{3}$ Department of Ophthalmology, \\ Birmingham Children's Hospital \\ NHS Foundation Trust, Birmingham, \\ ${ }^{4}$ Department of Ophthalmology, \\ Ninewells Hospital and Medical \\ School, Dundee, ${ }^{5}$ National Institute of \\ Health Research Biomedical Research \\ Centre, Moorfields Eye Hospital NHS \\ Foundation Trust and UCL Institute of \\ Ophthalmology, London, ${ }^{6}$ Centre for \\ Health and Social Care Improvement, \\ School of Health and Wellbeing, \\ University of Wolverhampton, \\ Wolverhampton, UK
}

Background: Ocular trauma is an important cause of visual morbidity in children worldwide. Pediatric ocular trauma accounts for up to one third of all ocular trauma admissions, with significant economic implications for health care providers. It is estimated that $90 \%$ of all ocular trauma is preventable. Development of strategies to reduce the incidence and severity of pediatric ocular trauma requires an understanding of the epidemiology of these injuries and their characteristics. This will enable appropriate targeting of resources toward prevention and allow effective service planning. At present, there is no standardized methodology for the collection of global cross-sectional data in pediatric ocular trauma, and the ability to undertake detailed epidemiological and health-economic analyses is limited. Furthermore, it is difficult to draw international comparisons in incidence, etiology, and outcomes of pediatric ocular trauma due to the range of published reporting criteria. This study describes two novel questionnaires for standardized data collection in pediatric ocular trauma, which can be adopted across a range of health care settings internationally.

Methods: Two standardized data collection questionnaires have been developed from previously reported templates. The first enables collection of demographic and incident data on serious pediatric ocular trauma requiring hospitalization, and the second enables follow-up outcome data collection. Both the questionnaires are designed to collect primarily categorical data in order to increase ease of completion and facilitate quantitative analysis. These questionnaires enable acquisition of standardized data on the incidence, etiology, and outcomes of pediatric ocular trauma.

Discussion: These questionnaires enable collection of standardized data and are designed for global use across all health care settings. Through prospective data collection, epidemiological trends can be determined, allowing health care providers to develop collaborative global preventive strategies. Furthermore, the same questionnaires may be used in future studies to draw comparisons with baseline data, allowing assessment of the efficacy of targeted preventative interventions.

Keywords: childhood eye injury, epidemiology, health economic analyses, international standardization, penetrating eye injury, perforating eye injury, prevention

\section{Background}

Ocular trauma in childhood is a significant cause of morbidity. Worldwide estimates suggest that ocular trauma is responsible for up to 280,000 hospital admissions annually in children aged $<15$ years. ${ }^{1}$ However, ocular trauma cases requiring hospital admission 
are thought to account for only $5 \%$ of all cases, ${ }^{2}$ which suggests that $\sim 6$ million children aged $<15$ years experience an episode of ocular trauma each year. Up to $24 \%$ of all patients admitted with ocular trauma have suffered a penetrating eye injury. ${ }^{3}$ Global estimates suggest that there are 3.9 million people living with bilateral visual loss and $>18$ million with unilateral visual loss due to ocular trauma. ${ }^{4}$

The significance of ocular trauma in childhood must not be underestimated. Since ocular injury is very rarely fatal, affected individuals must learn to live with the consequences of their injury for many years. Visual impairment can have a profound impact on activities of daily living, with marked socioeconomic implications for both the patient and the health care providers. ${ }^{5}$

Fortunately, up to $90 \%$ of all ocular trauma is thought to be preventable. ${ }^{6}$ Although the potential benefit of measures such as the use of simple eye protection have previously been discussed, the multifactorial nature of pediatric ocular trauma is likely to necessitate a more targeted approach to injury prevention, dependent on the local population. ${ }^{7,8}$ In order to implement targeted interventions to reduce the incidence of such injuries, it is first necessary to have a detailed understanding of the etiology, incidence, and outcomes of these injuries. Data collection in this field has been sporadic to date, with a lack of standardized, validated collection tools. As a result, the ability to draw meaningful comparisons between existing studies and the ability to extrapolate published data to target populations are currently limited. Detailed epidemiological and health-economic analysis has not been possible.

In order to address this shortcoming, two standardized data collection tools have been developed to enable collection of both incident and follow-up data from units managing pediatric ocular trauma. It is hoped that these questionnaires will be adopted internationally, in order to enable collection of data on a global scale, and facilitate implementation of preventative strategies.

\section{Methods}

First, a selection of generic key research aims are identified for any population-based study of pediatric ocular trauma, which should be considered useful outcomes in all future analyses regardless of geographical setting:

1. To determine the incidence of pediatric ocular trauma

2. To determine the demographic characteristics of injured children

3. To determine the causes/mechanisms and places of injury
4. To describe the clinical management of pediatric ocular trauma

5. To evaluate the visual outcome of injured children.

Having identified these aims, two data collection tools were designed: the first for incident data collection at initial presentation and the second to enable outcome data collection after a period of follow-up. Because of the success of previous UK-based studies, we developed our questionnaires from a previously published and validated template. ${ }^{9,10}$

Response categories for each questionnaire were informed by our previous large-scale literature review. ${ }^{1}$ Incident data comprised key features from patient history, initial clinical examination, and immediate management. Follow-up data comprised attendance status (under continuing follow-up, discharged, or lost-to-follow-up), progress (including treatment interventions and ocular sequelae of injury), and consequences (resulting visual impairment or low vision registration). Where possible, data input was restricted to categorical variables. This enabled rapid completion of the questionnaire by the reporting clinician and facilitated quantitative analysis of collected data. Free-text entry boxes were provided as a precaution in case of data falling outside of the pre-determined response categories.

It was intended that the requested information should be available from patient case notes with no additional tests or procedures requested. A minimum number of unique identifiers were included (hospital number, month and year of birth, gender, and ethnicity), in order to identify duplicate reports and to allow collection of follow-up data. These variables also allowed analysis of the potential relationship between the incidence of eye injury and other factors such as age, gender, and ethnicity.

Our incident and follow-up data collection questionnaires are shown in Supplementary material.

\section{BOSU POTS Data Collection UK}

The UK Paediatric Ocular Trauma Study (POTS) has employed these questionnaires to collect prospective data on pediatric ocular trauma in the UK. UK-based ophthalmologists have been asked to report incident data over a 12-month period, with follow-up data collection on each reported case after 12 months. This UK surveillance study has enabled us to identify the current incidence and pattern of serious pediatric ocular trauma that requires hospitalization in the UK and the resulting visual morbidity. The data collected will be used to recommend targeted preventive strategies, which can be evaluated by future comparisons with these baseline data. 
This study was conducted in association with the British Ophthalmological Surveillance Unit (BOSU), using their monthly reporting card scheme; this is an active surveillance system involving all UK consultant ophthalmologists. At the end of each month, BOSU sent out reporting cards to all UK ophthalmologists, in which they were asked to indicate whether they have seen a new case of pediatric ocular trauma. Ophthalmologists were requested to report any cases of ocular trauma involving children aged $\leq 16$ years, presenting in the preceding month that required hospital admission for observation, medical treatment or surgery, or day case admission for any procedure due to trauma. Ophthalmologists were requested to include both patients referred to them and patients they referred on to other ophthalmologists.

Reporting cards were returned to BOSU, who informed the investigator of each reported case with details of the reporting ophthalmologist (name, hospital, and hospital address). The incident questionnaire (Figure S1) was sent to the reporting ophthalmologists for data collection. To facilitate the return of the completed questionnaire, a selfaddressed envelope was included. The questionnaire was re-sent after 4-8 weeks if no response was received from the reporting ophthalmologist. After the third attempt, an effort was made to contact the ophthalmologist or the secretary directly through telephone call. For those cases in which incident data were collected, the follow-up questionnaire (Figure S2) was sent 12 months after the initial injury to collect follow-up data.

\section{Discussion}

We have developed two standardized data collection tools for use in epidemiologic studies of pediatric ocular trauma. The POTS questionnaires enable rapid and consistent data acquisition from patient records, requiring minimal effort on behalf of the reporting clinician, and impose no extra burden on the patient. These questionnaires are applicable to all pediatric populations, regardless of geographical location and have been successfully used as reporting tools in a UKbased surveillance study (results currently being analyzed). Widespread adoption of these questionnaires will enable detailed epidemiological and health-economic analyses of pediatric ocular trauma and allow direct comparisons to be made between patient cohorts on both a national and an international basis. Such analyses will facilitate the development of effective primary prevention strategies and allow accurate service planning to ensure that the highest standard of care can be offered to children in primary and secondary care settings worldwide.
The POTS questionnaires have been adapted from a published template; the Scottish Ocular Trauma Studies (SCOTS 1 and 2) $)^{9,10}$ have previously validated structured questionnaires for the collection of both incident and follow-up data, achieving impressive response rates and producing useful epidemiological analyses of the target population. Given the success of these studies, we chose to adopt a similar approach for the POTS questionnaires. The SCOTS questionnaires were developed for data collection from both pediatric and adult populations; in designing a pediatric-specific proforma, we were able to increase the amount of quantitative (categorical) data collected and minimize the amount of qualitative data generated, saving time both for the clinicians completing the proformas and researchers analyzing the collected data.

Development of strategies to reduce the incidence and severity of pediatric ocular trauma requires a thorough understanding of the underlying epidemiology and characteristics. This is dependent on the ability to collect robust and comprehensive data from the target population. Although there have been numerous previous studies gathering epidemiological data on pediatric ocular trauma, these have often been relatively small-scale, single-center cross-sectional studies from disparate populations, with each using a different template for data collection. ${ }^{1}$ The ability to extrapolate the findings of these studies to other groups is therefore limited. Furthermore, many of these studies could now be considered outdated. Effective service planning and development of strategies for targeted prevention is dependent on up-to-date data; we therefore suggest that data collection should be a continuous process with frequent re-audit of present service demands. Novel preventative interventions are unlikely to achieve their full potential when informed by historical data.

The POTS questionnaires address many of these shortcomings; they can be distributed nationally or internationally to enable rapid acquisition of a large volume of data and, if widely adopted, are a significant step toward international standardization of data collection templates. The design of the incident and follow-up questionnaires also facilitates re-audit and offers a method of continuous data acquisition.

Clinician-completed questionnaires are subject to a number of limitations, including reporting and recall biases, and may be limited by low response rates. Furthermore, there may be differences in the interpretation of response categories between clinicians, resulting in inconsistencies in data collected from different sources. However, the POTS collection questionnaires are intended for systemic, prospective 
data collection, ideally through active surveillance programs such as the BOSU reporting system. Such methods have previously been demonstrated to achieve higher response rates than retrospective or passive surveillance methods, with reduced recall and reporting bias. ${ }^{11,12}$ Throughout the course of data collection for the BOSU POTS surveillance study, the senior investigator (FS) also monitored all returned questionnaires, making direct contact with clinicians through telephone both to encourage completion and to clarify inconsistencies in collected data where appropriate.

Although these questionnaires have been developed primarily for use as paper-based data collection tools, they could easily be adapted to other formats, for example, electronic forms that could be completed and returned through email. The use of alternate formats may further increase the response rates.

We share a common ambition across the ophthalmic research community; that is, to reduce the incidence and morbidity of pediatric ocular trauma and improve visual outcomes for children. We offer the POTS questionnaires to the international community in the hope that the questionnaires will be widely adopted, resulting in increased reporting of epidemiological trends in pediatric ocular trauma and subsequent introduction of effective primary prevention interventions.

\section{Ethical approval}

This study was approved by the Cambridge South Research Ethics Committee, Cambridge, UK. R\&D approval was granted by Birmingham Children's Hospital Research and Development Department, Birmingham, UK.

\section{Acknowledgment}

The authors wish to thank the BOSU, in particular Mr Barny Foot for his advice at various stages of this project's inception. We also acknowledge the BOSU review committee members for their contribution to the design of POTS data collection questionnaires. This study was supported by a bursary from the BOSU.

\section{Author contributions}

All authors made substantial contributions to conception and design, acquisition of data, or analysis and interpretation of data; took part in drafting the article or revising it critically for important intellectual content; gave final approval of the version to be published; and agree to be accountable for all aspects of the work.

\section{Disclosure}

The authors report no conflicts of interest in this work.

\section{References}

1. Abbott J, Shah P. The epidemiology and etiology of pediatric ocular trauma. Surv Ophthalmol. 2013;58(5):476-485.

2. May DR, Kuhn FP, Morris RE, et al. The epidemiology of serious eye injuries from the United States Eye Injury Registry. Graefes Arch Clin Exp Ophthalmol. 2000;238(2):153-157.

3. Strahlman E, Elman M, Daub E, Baker S. Causes of pediatric eye injuries. A population-based study. Arch Ophthalmol. 1990;108(4):603-606.

4. Négrel AD, Thylefors B. The global impact of eye injuries. Ophthalmic Epidemiol. 1998;5(3):143-169.

5. Rahi JS, Cumberland PM, Peckham CS. Visual function in workingage adults: early life influences and associations with health and social outcomes. Ophthalmology. 2009;116(10):1866-1871.

6. Pizzarello LD. Ocular trauma: time for action. Ophthalmic Epidemiol. 1998;5(3):115-116.

7. Philip S, Hoskin A. Children's protective eyewear: the challenges and the way forward. Med J Aust. 2014;201(2):87-88.

8. Hoskin A, Philip S, Yardley A, Mackey D. Eye injury prevention for the paediatric population. Asia Pac J Ophthalmol. 2016;5:202-211.

9. Desai P, MacEwen CJ, Baines P, Minassian DC. Incidence of cases of ocular trauma admitted to hospital and incidence of blinding outcome. Br J Ophthalmol. 1996;80(7):592-596.

10. Morris DS, Willis S, Minassian D, Foot B, Desai P, MacEwen CJ. The incidence of serious eye injury in Scotland: a prospective study. Eye. 2014;28(1):34-40.

11. Thacker SB, Redmond S, Rothenberg RB, Spitz SB, Choi K, White MC. A controlled trial of disease surveillance strategies. Am J Prev Med. 1986;2(6):345-350.

12. Vogt RL, LaRue D, Klaucke DN, Jillson DA. Comparison of an active and passive surveillance system of primary care providers for hepatitis, measles, rubella, and salmonellosis in Vermont. Am J Public Health. 1983;73(7):795-797.
Clinical Ophthalmology

\section{Publish your work in this journal}

Clinical Ophthalmology is an international, peer-reviewed journal covering all subspecialties within ophthalmology. Key topics include: Optometry; Visual science; Pharmacology and drug therapy in eye diseases; Basic Sciences; Primary and Secondary eye care; Patient Safety and Quality of Care Improvements. This journal is indexed on

\section{Dovepress}

PubMed Central and CAS, and is the official journal of The Society of Clinical Ophthalmology (SCO). The manuscript management system is completely online and includes a very quick and fair peer-review system, which is all easy to use. Visit http://www.dovepress.com/ testimonials.php to read real quotes from published authors. 